MATHEMATICS OF COMPUTATION

Volume 76, Number 258, April 2007, Pages 931-945

S 0025-5718(06)01920-X

Article electronically published on November 27, 2006

\title{
UNIVARIATE SPLINES: EQUIVALENCE OF MODULI OF SMOOTHNESS AND APPLICATIONS
}

\author{
KIRILL A. KOPOTUN
}

\begin{abstract}
Several results on equivalence of moduli of smoothness of univariate splines are obtained. For example, it is shown that, for any $1 \leq k \leq r+1$, $0 \leq m \leq r-1$, and $1 \leq p \leq \infty$, the inequality $n^{-\nu} \omega_{k-\nu}\left(s^{(\bar{\nu})}, n^{-1}\right)_{p} \sim$ $\omega_{k}\left(s, n^{-1}\right)_{p}, 1 \leq \nu \leq \min \{k, m+1\}$, is satisfied, where $s \in \mathbb{C}^{m}[-1,1]$ is a piecewise polynomial of degree $\leq r$ on a quasi-uniform (i.e., the ratio of lengths of the largest and the smallest intervals is bounded by a constant) partition of an interval. Similar results for Chebyshev partitions and weighted Ditzian-Totik moduli of smoothness are also obtained. These results yield simple new constructions and allow considerable simplification of various known proofs in the area of constrained approximation by polynomials and splines.
\end{abstract}

\section{INTRODUCTION AND MAIN RESULTS}

This paper investigates properties of various moduli of smoothness of univariate piecewise polynomial functions (splines). It turns out that the classical estimates of moduli of smoothness of functions via appropriate moduli of their derivatives can be reversed if the functions being considered are splines. This observation has several applications, and, in particular, yields simple new constructions and allows considerable simplification of various known proofs of Jackson type inequalities for constrained approximation by polynomials and smooth splines (see Section 5 on "Applications" for more detail).

Let $\mathcal{S}_{r}\left(\mathbf{z}_{n}\right)$ be the space of all piecewise polynomial functions of degree $r$ (order $r+1)$ with the knots $\mathbf{z}_{n}:=\left(z_{i}\right)_{i=0}^{n},-1=: z_{0}<z_{1}<\cdots<z_{n-1}<z_{n}:=1$. In other words, we say that $s \in \mathcal{S}_{r}\left(\mathbf{z}_{n}\right)$ if, on each interval $\left(z_{i}, z_{i+1}\right), 0 \leq i \leq$ $n-1, s$ is in $\Pi_{r}$, where $\Pi_{r}$ denotes the space of algebraic polynomials of degree $\leq r$. Usually, piecewise polynomials from $\mathcal{S}_{r}\left(\mathbf{z}_{n}\right)$ are called "splines" (or "splines of minimal defect") if they possess continuous $(r-1)$ st derivatives. In this paper, we refer to all piecewise polynomials from $\mathcal{S}_{r}\left(\mathbf{z}_{n}\right)$ as splines. In other words, we do not have any a priori assumptions on their smoothness or even continuity at the knots $\mathbf{z}_{n}$. We also assume that a spline and its derivatives are defined at the knots by continuity, if possible, and not defined otherwise, and that all derivatives of splines from $\mathcal{S}_{r}\left(\mathbf{z}_{n}\right)$ are understood "pointwise" (for example, the first derivative of a characteristic function of an interval $[a, b]$ is identically 0 except for the points

Received by the editor June 1, 2005 and, in revised form, August 25, 2005.

2000 Mathematics Subject Classification. Primary 65D07, 41A15, 26A15; Secondary 41A10, 41A25, 41A29.

Key words and phrases. Univariate splines, moduli of smoothness, degree of approximation, Jackson type estimates, polynomial and spline approximation.

The author was supported in part by NSERC of Canada.

(c)2006 American Mathematical Society Reverts to public domain 28 years from publication 
$a$ and $b$, where it is undefined). We emphasize that, with these assumptions, if $s \in \mathcal{S}_{r}\left(\mathbf{z}_{n}\right)$ and $m \in \mathbb{N}$, then $s^{(m)} \in \mathcal{S}_{\max \{r-m, 0\}}\left(\mathbf{z}_{n}\right)$ and, in fact, $s^{(m)}=0$ a.e. if $m \geq r+1$.

As usual, $\mathbb{L}_{p}(J), 0<p \leq \infty$, denotes the space of all measurable functions $f$ on $J$ such that $\|f\|_{\mathbb{L}_{p}(J)}<\infty$, where $\|f\|_{\mathbb{L}_{p}(J)}:=\left(\int_{J}|f(x)|^{p} d x\right)^{1 / p}$ if $p<\infty$, and $\|f\|_{\mathbb{L}_{\infty}(J)}:=\operatorname{ess}_{\sup _{x \in J}}|f(x)|$. We also denote $\|f\|_{p}:=\|f\|_{\mathbb{L}_{p}[-1,1]}$. It is well known that $\|\cdot\|_{\mathbb{L}_{p}(J)}$ is a norm (and $\mathbb{L}_{p}(J)$ is a Banach space) if $1 \leq p \leq \infty$, and that it is a quasi-norm if $0<p<1$.

For $k \in \mathbb{N}_{0}$, let

$$
\Delta_{h}^{k}(f, x, J):= \begin{cases}\sum_{i=0}^{k}\left(\begin{array}{c}
k \\
i
\end{array}\right)(-1)^{k-i} f(x-k h / 2+i h), & \text { if } x \pm k h / 2 \in J, \\
0, & \text { otherwise, }\end{cases}
$$

be the $k$ th symmetric difference, and let $\Delta_{h}^{k}(f, x):=\Delta_{h}^{k}(f, x,[-1,1])$. The $k$ th modulus of smoothness of a function $f \in \mathbb{L}_{p}(J)$ is defined by

$$
\omega_{k}(f, t, J)_{p}:=\sup _{0<h \leq t}\left\|\Delta_{h}^{k}(f, \cdot, J)\right\|_{\mathbb{L}_{p}(J)}
$$

and we also denote

$$
\omega_{k}(f, J)_{p}:=\omega_{k}(f,|J|, J)_{p} \quad \text { and } \quad \omega_{k}(f, t)_{p}:=\omega_{k}(f, t,[-1,1])_{p} .
$$

Note that $\Delta_{h}^{0}(f, x, J):=f(x)$ and, hence, $\omega_{0}(f, t, J)_{p}:=\|f\|_{\mathbb{L}_{p}(J)}$.

For a partition $\mathbf{z}_{n}:=\left\{z_{0}, \ldots, z_{n} \mid-1=: z_{0}<z_{1}<\cdots<z_{n}:=1\right\}$ of the interval $[-1,1]$, denote the scale of the partition $\mathbf{z}_{n}$ by

$$
\vartheta:=\vartheta\left(\mathbf{z}_{n}\right):=\max _{0 \leq j \leq n-1} \frac{\left|J_{j \pm 1}\right|}{\left|J_{j}\right|}
$$

where $J_{j}:=\left[z_{j}, z_{j+1}\right]$ with $z_{j}:=-1, j<0$, and $z_{j}:=1, j>n$, and $|J|:=$ meas $J$.

Throughout this paper, $c\left(\gamma_{1}, \gamma_{2}, \ldots\right)$ denote positive constants which depend only on the parameters $\gamma_{1}, \gamma_{2}, \ldots$ (note that $c(p, \ldots)$ depends on $p$ only as $p \rightarrow 0$ ) and which may be different on different occurrences. At the same time, $c_{i}, i=$ $0,1, \ldots, 7$, denote positive constants which are fixed throughout the paper.

Theorem 1.1 (Local estimates). Let $s \in \mathcal{S}_{r}\left(\mathbf{z}_{n}\right), r \in \mathbb{N}$, and $J=\left[z_{\mu_{1}}, z_{\mu_{2}}\right]$ with $\mu_{2}-\mu_{1} \leq c_{0}$ for some constant $c_{0}$. Then, for any $1 \leq k \leq r+1$ and $0<p \leq \infty$, we have

$$
\omega_{k-\nu}\left(s^{(\nu)}, J\right)_{p} \leq c\left(r, \vartheta, c_{0}, p\right)|J|^{-\nu} \omega_{k}(s, J)_{p},
$$

for all $\nu=1, \ldots, k$.

We say that $A$ is equivalent to $B$ and write $A \sim B$ if there exists a positive constant $c$ such that $c^{-1} A \leq B \leq c A$. We refer to this constant $c$ as an equivalence constant.

It is well known that inequality (1.2) can be reversed in the case $p \geq 1$ if $s^{(\nu-1)}$ is continuous (see (2.3)). Together with Theorem 1.1 this immediately implies the following.

Corollary 1.2 (Local estimates: Equivalence of moduli of smoothness). Let $s \in$ $\mathcal{S}_{r}\left(\mathbf{z}_{n}\right) \cap \mathbb{C}^{m}[-1,1], r \in \mathbb{N}, 0 \leq m \leq r-1$, and $J=\left[z_{\mu_{1}}, z_{\mu_{2}}\right]$ with $\mu_{2}-\mu_{1} \leq c_{0}$ for 
some constant $c_{0}$. Then, for any $1 \leq k \leq r+1$ and $1 \leq p \leq \infty$, we have

$$
|J|^{\nu} \omega_{k-\nu}\left(s^{(\nu)}, J\right)_{p} \sim \omega_{k}(s, J)_{p}, \quad 1 \leq \nu \leq \min \{k, m+1\} .
$$

Equivalence constants above depend only on $r, \vartheta$ and $c_{0}$.

Remark 1.3. Inequality (1.2) can also be reversed in the case $0<p<1$. This implies that all results stated in this paper for $1 \leq p \leq \infty$ have their analogs in the case $0<p<1$ as well. Proofs (some of which are rather involved) as well as corresponding corollaries on equivalence of (global) moduli of smoothness in the case $0<p<1$ will appear elsewhere*

We are now ready to state several corollaries of Theorem 1.1 involving global moduli of smoothness of splines on various partitions. First, we consider the case when $\mathbf{z}_{n}$ is uniform or, more generally, quasi-uniform.

Suppose that $\delta_{\max }:=\delta_{\max }\left(\mathbf{z}_{n}\right):=\max _{0 \leq j \leq n-1}\left|J_{j}\right|$ and $\delta_{\min }:=\delta_{\min }\left(\mathbf{z}_{n}\right):=$ $\min _{0 \leq j \leq n-1}\left|J_{j}\right|$. We say that $\mathbf{z}_{n}$ is $\Delta$-quasi-uniform if $\Delta:=\delta_{\max } / \delta_{\min }$ is bounded by a constant independent of $n$, and denote such partition by $\mathbf{u}_{n}^{\Delta}$. Note that the 1-quasi-uniform partition $\mathbf{u}_{n}:=\mathbf{u}_{n}^{1}$ is just the uniform partition of $[-1,1]$ into $n$ subintervals of equal lengths.

If $\mathbf{z}_{n}=\mathbf{u}_{n}^{\Delta}$, then clearly $2 /(n \Delta) \leq \delta_{\min } \leq 2 / n \leq \delta_{\max } \leq 2 \Delta / n$, and $\vartheta\left(\mathbf{z}_{n}\right) \leq \Delta$. Therefore, $\delta_{\min } \sim \delta_{\max } \sim n^{-1}$ with equivalence constants depending only on $\Delta$.

Theorem 1.4 (Quasi-uniform partition). Let $\mathbf{u}_{n}^{\Delta}, n \in \mathbb{N}$, be a $\Delta$-quasi-uniform partition of $[-1,1]$, and let $s \in \mathcal{S}_{r}\left(\mathbf{u}_{n}^{\Delta}\right), r \in \mathbb{N}$. Then, for any $1 \leq k \leq r+1$ and $0<p \leq \infty$, we have

$$
\omega_{k-\nu}\left(s^{(\nu)}, n^{-1}\right)_{p} \leq c(r, \Delta, p) n^{\nu} \omega_{k}\left(s, n^{-1}\right)_{p},
$$

for all $\nu=1, \ldots, k$.

Together with the inequality (2.3) this now implies the following result on equivalence of classical moduli of smoothness of splines on quasi-uniform partitions.

Corollary 1.5 (Quasi-uniform partition: Equivalence of moduli of smoothness). Let $\mathbf{u}_{n}^{\Delta}, n \in \mathbb{N}$, be a $\Delta$-quasi-uniform partition of $[-1,1]$, and let $s \in \mathcal{S}_{r}\left(\mathbf{u}_{n}^{\Delta}\right) \cap$ $\mathbb{C}^{m}[-1,1], r \in \mathbb{N}, 0 \leq m \leq r-1$. Then, for any $1 \leq k \leq r+1$ and $1 \leq p \leq \infty$, we have

$$
n^{-\nu} \omega_{k-\nu}\left(s^{(\nu)}, n^{-1}\right)_{p} \sim \omega_{k}\left(s, n^{-1}\right)_{p}, \quad 1 \leq \nu \leq \min \{k, m+1\} .
$$

Equivalence constants above depend only on $r$ and $\Delta$.

Remark 1.6. For $m=k-1$, (1.4) was proved by $\mathrm{Hu}$ and $\mathrm{Yu}[12$ and by $\mathrm{Hu}$, for uniform and quasi-uniform partitions, respectively. In fact, if we set $m=k-1$, then Corollary [1.5 (with an additional restriction $k \leq r$ ) becomes Theorem 1 in [10. (and so, in the case $k \leq m+1 \leq r$, it follows from [10, Theorem 1]). Also, in the case $k=r+1$ and $m=r-1$, Corollary 1.5 follows from Theorem 2 of [10] (where (1.4) was proved for all $k \geq r+1$ in the case $m=r-1$ ).

The weighted Ditzian-Totik (DT-) $k$ th modulus of smoothness of a function $f \in \mathbb{L}_{p}[-1,1], 0<p \leq \infty$, is defined by

$$
\omega_{k, \nu}^{\varphi}(f, t)_{p}:=\sup _{0<h \leq t}\left\|\varphi(\cdot)^{\nu} \Delta_{h \varphi(\cdot)}^{k}(f, \cdot)\right\|_{p},
$$

${ }^{*} \mathrm{~K}$. A. Kopotun, On equivalence of moduli of smoothness of splines in $L_{p}, 0<p<1$, J. Approx. Theory 143 (2006), no. 1, 36-43. 
where $\varphi(x):=\sqrt{1-x^{2}}$. If $\nu=0$, then

$$
\omega_{k}^{\varphi}(f, t)_{p}:=\omega_{k, 0}^{\varphi}(f, t)_{p}=\sup _{0<h \leq t}\left\|\Delta_{h \varphi(\cdot)}^{k}(f, \cdot)\right\|_{p}
$$

is the usual DT-modulus [9]. Also, note that

$$
\omega_{0, \nu}^{\varphi}(f, t)_{p}:=\left\|\varphi^{\nu} f\right\|_{p} .
$$

Remark 1.7. It is possible that $\varphi^{\nu} f \in \mathbb{L}_{p}[-1,1]$ and $\omega_{k, \nu}^{\varphi}(f, t)_{p}=\infty$. For example, this is the case for $f(x)=(1+x)^{-1}, k=\nu=1$ and $1<p<2$.

We now obtain several corollaries for splines with Chebyshev knots. We say that $\mathbf{z}_{n}$ is a Chebyshev partition (and $z_{i}$ 's are Chebyshev knots) if $\mathbf{z}_{n}=\mathbf{t}_{n}:=\left(t_{i}\right)_{i=0}^{n}$, where $t_{i}:=\cos \left(\frac{(n-i) \pi}{n}\right), 0 \leq i \leq n$.

Theorem 1.8 (Chebyshev knots). Let $\mathbf{t}_{n}$ be a Chebyshev partition of $[-1,1]$. If $s \in \mathcal{S}_{r}\left(\mathbf{t}_{n}\right), r \in \mathbb{N}$, then, for any $1 \leq k \leq r+1$ and $0<p \leq \infty$, we have

$$
\omega_{k-\nu, \nu}^{\varphi}\left(s^{(\nu)}, n^{-1}\right)_{p} \leq c(r, p) n^{\nu} \omega_{k}^{\varphi}\left(s, n^{-1}\right)_{p},
$$

for all $1 \leq \nu \leq k$.

The following result now immediately follows from Theorems 1.8 and 6.1 (in the case $k=\nu$, see also [9, (2.4.4)]).

Corollary 1.9 (Chebyshev knots: Equivalence of moduli for $1 \leq p \leq \infty$ ). Let $s \in \mathcal{S}_{r}\left(\mathbf{t}_{n}\right) \cap \mathbb{C}^{m}[-1,1], r \in \mathbb{N}, 0 \leq m \leq r-1$. Then, for any $1 \leq k \leq r+1$, $1 \leq \nu \leq \min \{k, m+1\}$ and $1 \leq p \leq \infty$, we have

$$
n^{-\nu} \omega_{k-\nu, \nu}^{\varphi}\left(s^{(\nu)}, n^{-1}\right)_{p} \sim \omega_{k}^{\varphi}\left(s, n^{-1}\right)_{p} .
$$

Equivalence constants above depend only on $r$.

The paper is organized as follows. In Section 2, we recall some classical properties of the moduli of smoothness and algebraic polynomials, as well as discuss some auxiliary results for Chebyshev partitions. Theorems 1.1, 1.4 and 1.8 are proved in Section 3. General applications for constrained approximation by polynomials and smooth splines are given in Section 4, and Section 5 is devoted to applications in the area of monotone and convex approximation. Finally, an important property of the weighted DT-moduli needed to obtain Corollary 1.9 is established in Section 6 .

\section{Auxiliary Results}

2.1. Classical moduli of smoothness. The following properties of the moduli of smoothness are well known (see, e.g., [8,16]).

(i) For $f \in \mathbb{L}_{p}(J), 0<p \leq \infty$, we have

and

$$
\omega_{k+1}(f, t, J)_{p} \leq 2^{\max \{1,1 / p\}} \omega_{k}(f, t, J)_{p}, \quad k \in \mathbb{N},
$$

$$
\omega_{k}(f, \lambda t, J)_{p} \leq c(k, p)(\lambda+1)^{k-1+\max \{1,1 / p\}} \omega_{k}(f, t, J)_{p}, \quad \lambda>0 .
$$

(ii) If $f^{(\nu-1)} \in A C(J), \nu \in \mathbb{N}$, and $f^{(\nu)} \in \mathbb{L}_{p}(J), 1 \leq p \leq \infty$, then

$$
\omega_{k+\nu}(f, t, J)_{p} \leq t^{\nu} \omega_{k}\left(f^{(\nu)}, t, J\right)_{p} .
$$

Note that this inequality is no longer true in general if $0<p<1$. 
(iii) If $f \in \mathbb{L}_{p}[a, b], 0<p<\infty$, and $\gamma \in \mathbb{N}$, then (see [16, Lemma 7.2])

$$
\omega_{\gamma}(f, \delta,[a, b])_{p}^{p} \leq \frac{c(\gamma, p)}{\delta} \int_{0}^{\delta} \int_{a}^{b}\left|\Delta_{h}^{\gamma}(f, x,[a, b])\right|^{p} d x d h .
$$

(iv) Suppose that $f \in \mathbb{L}_{p}[-1,1], 0<p<\infty$, and $k, \mu \in \mathbb{N}$. Then (see, e.g., [7, 16]),

$$
\sum_{j=0}^{n-\mu-1} \omega_{k}\left(f, \bigcup_{i=j}^{j+\mu} J_{i}\right)_{p}^{p} \leq \begin{cases}c(k, \Delta, \mu, p) \omega_{k}\left(f, n^{-1}\right)_{p}^{p}, & \text { if } \mathbf{z}_{n}=\mathbf{u}_{n}^{\Delta}, \\ c(k, \mu, p) \omega_{k}^{\varphi}\left(f, n^{-1}\right)_{p}^{p}, & \text { if } \mathbf{z}_{n}=\mathbf{t}_{n} .\end{cases}
$$

2.2. Algebraic polynomials. We now recall several well known facts about algebraic polynomials which are frequently used in the proofs.

Lemma 2.1 (Whitney's inequality). For any $f \in \mathbb{L}_{p}[a, b], 0<p \leq \infty$, there exists $q_{k-1} \in \Pi_{k-1}$ such that

$$
\left\|f-q_{k-1}\right\|_{\mathbb{L}_{p}[a, b]} \leq C \omega_{k}(f,[a, b])_{p} .
$$

Lemma 2.2. For any polynomial $q_{r} \in \Pi_{r}, 0<p \leq \infty$, and intervals $I$ and $J$ such that $I \subseteq J$, we have

$$
|J|^{1 / p}\left\|q_{r}^{(\nu)}\right\|_{\mathbb{L}_{\infty}(J)} \sim\left\|q_{r}^{(\nu)}\right\|_{\mathbb{L}_{p}(J)} \leq c(r,|J| /|I|, p)|J|^{-\nu}\left\|q_{r}\right\|_{\mathbb{L}_{p}(I)}, \quad 0 \leq \nu \leq r
$$

where equivalence constants depend only on $r$ and $p($ as $p \rightarrow 0$ ).

2.3. Chebyshev partitions. Suppose that $\mathbf{t}_{n}$ is a Chebyshev partition, recall that $J_{j}:=\left[t_{j}, t_{j+1}\right], 0 \leq j \leq n-1$, and denote

$$
\mathfrak{D}_{\delta}:=\{x|1-\delta \varphi(x) / 2 \geq| x \mid\} \backslash\{ \pm 1\}=\left\{x|| x \mid \leq \frac{4-\delta^{2}}{4+\delta^{2}}\right\} .
$$

Observe that $\mathfrak{D}_{\delta}=\emptyset$ if $\delta>2$, and note that $\Delta_{h \varphi(x)}^{k}(f, x)$ is defined to be identically 0 if $x \notin \mathfrak{D}_{k h}$.

It is a simple exercise to show that, for

$$
\begin{gathered}
\alpha:=\frac{\min \left\{\mu_{1}+\mu_{2}, 2 n-\mu_{1}-\mu_{2}\right\}}{\min \left\{2 \mu_{1}+1,2 n-2 \mu_{1}-1\right\}}, \\
\frac{4 \alpha}{\pi^{2}}\left|\mu_{1}-\mu_{2}\right|\left|J_{\mu_{1}}\right| \leq\left|t_{\mu_{1}}-t_{\mu_{2}}\right| \leq \frac{\pi^{2} \alpha}{4}\left|\mu_{1}-\mu_{2}\right|\left|J_{\mu_{1}}\right|, \quad 0 \leq \mu_{1} \leq n-1, \quad 0 \leq \mu_{2} \leq n .
\end{gathered}
$$

Therefore, for large $n, x \in J_{j} \cap \mathfrak{D}_{m h}$ and $0<h \leq n^{-1}$, since $\varphi(x) \leq n\left|J_{j}\right|$ (see, e.g., [17, (17.4)]), we have

$$
\left\{x+\left(i-\frac{m}{2}\right) h \varphi(x)\right\}_{i=0}^{m} \subset\left[t_{j}-\frac{m\left|J_{j}\right|}{2}, t_{j+1}+\frac{m\left|J_{j}\right|}{2}\right] \cap[-1,1] \subset \mathfrak{I}_{j},
$$

where $\mathfrak{I}_{j}:=\mathfrak{I}_{j, m}:=\left[t_{j-3 m}, t_{j+4+3 m}\right]$ (recall that $t_{i}:=-1$ for $i<0$, and $t_{i}:=1$ for $i>n)$.

\section{Proofs of the MAIN RESUlts}

In all proofs in this section, we only consider the case $0<p<\infty$ since, for $p=\infty$, considerations are similar and, in fact, proofs are simpler. 
3.1. Proof of Theorem 1.1. Note that if $s$ were assumed to be sufficiently smooth we could reduce the proof of (1.2) to the case $\nu=k$ using the inequality $\omega_{k-\nu}\left(s^{(\nu)}, J\right)_{p} \leq c|J|^{k-\nu}\left\|s^{(k)}\right\|_{\mathbb{L}_{p}(J)}$. However, this inequality is not true for nonsmooth functions (a characteristic function of an interval is an obvious counterexample), and so this approach is not possible.

Everywhere in this proof, $q_{k-1}$ denotes a polynomial of degree $\leq k-1$ such that $\left\|s-q_{k-1}\right\|_{\mathbb{L}_{p}(J)} \leq c \omega_{k}(s, J)_{p}$ (existence of such $q_{k-1}$ is guaranteed by Whitney's inequality (2.6) ). For any $1 \leq \nu \leq k$, denoting $\mathfrak{s}_{j}:=\left.s\right|_{J_{j}}$ and using (2.7), we have

$$
\begin{aligned}
& \left|\Delta_{h}^{k-\nu}\left(s^{(\nu)}, x, J\right)\right|=\left|\Delta_{h}^{k-\nu}\left(s^{(\nu)}-q_{k-1}^{(\nu)}, x, J\right)\right| \leq 2^{k-\nu}\left\|s^{(\nu)}-q_{k-1}^{(\nu)}\right\|_{\mathbb{L}_{\infty}(J)} \\
& \quad \leq c(k) \max _{\mu_{1} \leq j \leq \mu_{2}-1}|| \mathfrak{s}_{j}^{(\nu)}-q_{k-1}^{(\nu)} \|_{\mathbb{L}_{\infty}\left(J_{j}\right)} \\
& \quad \leq c(r, p) \max _{\mu_{1} \leq j \leq \mu_{2}-1}\left|J_{j}\right|^{-\nu-1 / p}\left\|\mathfrak{s}_{j}-q_{k-1}\right\|_{\mathbb{L}_{p}\left(J_{j}\right)} \\
& \quad \leq c\left(r, \vartheta, c_{0}, p\right)|J|^{-\nu-1 / p}\left\|s-q_{k-1}\right\|_{\mathbb{L}_{p}(J)} \leq c\left(r, \vartheta, c_{0}, p\right)|J|^{-\nu-1 / p} \omega_{k}(s, J)_{p},
\end{aligned}
$$

which immediately implies (1.2).

3.2. Quasi-uniform partition: Proof of Theorem 1.4. Since $\mathbf{u}_{n}^{\Delta}$ is $\Delta$-quasiuniform, $\delta_{\max } \sim \delta_{\min } \sim\left|J_{j}\right| \sim n^{-1}$ for all $0 \leq j \leq n-1$ with equivalence constants depending only on $\Delta$. Using (2.2) we have

$$
\omega_{k-\nu}\left(s^{(\nu)}, n^{-1}\right)_{p}=\omega_{k-\nu}\left(s^{(\nu)}, \frac{k}{n \delta_{\min }} \cdot \frac{\delta_{\min }}{k}\right)_{p} \leq c(k, \Delta, p) \omega_{k-\nu}\left(s^{(\nu)}, \delta_{\min } / k\right)_{p} .
$$

Now, for any $0<h<\delta_{\min } / k$ and $x \in J_{j}, 0 \leq j \leq n-1$, such that $\Delta_{h}^{k-\nu}\left(s^{(\nu)}, x\right) \neq 0$, all points $x-(k-\nu) h / 2+i h, 0 \leq i \leq k-\nu$, are either in $J_{j-1} \cup J_{j}$ or $J_{j} \cup J_{j+1}$. Therefore,

$$
\begin{aligned}
& \omega_{k-\nu}\left(s^{(\nu)}, \delta_{\min } / k\right)_{p}^{p}=\sup _{0<h \leq \delta_{\min } / k}\left\|\Delta_{h}^{k-\nu}\left(s^{(\nu)}, \cdot\right)\right\|_{p}^{p} \\
& =\sup _{0<h \leq \delta_{\min } / k} \sum_{j=0}^{n-1}\left\|\Delta_{h}^{k-\nu}\left(s^{(\nu)}, \cdot\right)\right\|_{\mathbb{L}_{p}\left(J_{j}\right)}^{p} \\
& \leq \quad 2 \sup _{0<h \leq \delta_{\min } / k} \sum_{j=1}^{n-1}\left\|\Delta_{h}^{k-\nu}\left(s^{(\nu)}, \cdot, J_{j-1} \cup J_{j}\right)\right\|_{\mathbb{L}_{p}\left(J_{j-1} \cup J_{j}\right)}^{p} \\
& \leq 2 \sum_{j=1}^{n-1} \omega_{k-\nu}\left(s^{(\nu)}, J_{j-1} \cup J_{j}\right)_{p}^{p} \\
& \leq c(r, \Delta, p) \sum_{j=1}^{n-1}\left|J_{j-1} \cup J_{j}\right|^{-p \nu} \omega_{k}\left(s, J_{j-1} \cup J_{j}\right)_{p}^{p} \\
& \leq c(r, \Delta, p) n^{p \nu} \sum_{j=1}^{n-1} \omega_{k}\left(s, J_{j-1} \cup J_{j}\right)_{p}^{p} \leq c(r, \Delta, p) n^{p \nu} \omega_{k}\left(s, n^{-1}\right)_{p}^{p},
\end{aligned}
$$

where the last inequality follows from (2.5). 
3.3. Chebyshev knots: Proof of Theorem 1.8. Recall from Section 2 that $\mathfrak{I}_{j, k-\nu}=\left[t_{j-3(k-\nu)}, t_{j+4+3(k-\nu)}\right]$, denote $\mathfrak{I}_{j}:=\mathfrak{I}_{j, k-\nu}$, and, for each $0 \leq j \leq n-1$, let $\mathfrak{q}_{j} \in \Pi_{k-\nu-1}$ be such that $\left\|s^{(\nu)}-\mathfrak{q}_{j}\right\|_{\mathbb{L}_{p}\left(\mathfrak{I}_{j}\right)} \leq c \omega_{k-\nu}\left(s^{(\nu)}, \mathfrak{I}_{j}\right)_{p}\left(\mathfrak{q}_{j}\right.$ exists by Lemma 2.1, and we assume that $\mathfrak{q}_{j} \equiv 0$ if $\nu=k$ ). Then, using Theorem 1.1 and the inequality $\varphi(x) \leq n\left|J_{j}\right|, x \in J_{j}$, we have

$$
\begin{aligned}
& \omega_{k-\nu, \nu}^{\varphi}\left(s^{(\nu)}, n^{-1}\right)_{p}^{p}=\sup _{0<h \leq n^{-1}}\left\|\varphi^{\nu} \Delta_{h \varphi}^{k-\nu}\left(s^{(\nu)}, \cdot\right)\right\|_{p}^{p} \\
& =\sup _{0<h \leq n^{-1}} \sum_{j=0}^{n-1}\left\|\varphi^{\nu} \Delta_{h \varphi}^{k-\nu}\left(s^{(\nu)}, \cdot\right)\right\|_{\mathbb{L}_{p}\left(J_{j}\right)}^{p} \\
& \leq \sum_{j=0}^{n-1} n^{\nu p}\left|J_{j}\right|^{\nu p} \sup _{0<h \leq n^{-1}}\left\|\Delta_{h \varphi}^{k-\nu}\left(s^{(\nu)}-\mathfrak{q}_{j}, \cdot\right)\right\|_{\mathbb{L}_{p}\left(J_{j}\right)}^{p} \\
& \leq c(k, p) n^{\nu p} \sum_{j=0}^{n-1}\left|J_{j}\right|^{\nu p}\left\|s^{(\nu)}-\mathfrak{q}_{j}\right\|_{\mathbb{L}_{p}\left(\mathfrak{I}_{j}\right)}^{p} \\
& \leq c(k, p) n^{\nu p} \sum_{j=0}^{n-1}\left|J_{j}\right|^{\nu p} \omega_{k-\nu}\left(s^{(\nu)}, \mathfrak{I}_{j}\right)_{p}^{p} \\
& \leq c(r, p) n^{\nu p} \sum_{j=0}^{n-1} \omega_{k}\left(s, \mathfrak{I}_{j}\right)_{p}^{p} \leq c(r, p) n^{\nu p} \omega_{k}^{\varphi}\left(s, n^{-1}\right)_{p}^{p},
\end{aligned}
$$

where the last inequality follows from (2.5).

\section{Constrained approximation by polynomials and Smooth splines}

In this section, it is convenient to state the results simultaneously for the quasiuniform and Chebyshev partitions of $[-1,1]$, and so we need the following notation:

$$
\mathrm{w}_{m}\left(g^{(\nu)}, t\right)_{p}:= \begin{cases}\omega_{m}\left(g^{(\nu)}, t\right)_{p}, & \text { if } \mathbf{z}_{n}=\mathbf{u}_{n}^{\Delta}, \\ \omega_{m, \nu}^{\varphi}\left(g^{(\nu)}, t\right)_{p}, & \text { if } \mathbf{z}_{n}=\mathbf{t}_{n}\end{cases}
$$

Let $\mathcal{M}$ be a fixed class of functions, which we refer to as "constraints class". For example, $\mathcal{M}$ could be the class of all monotone, or all convex, or all $k$-monotone functions, or the class of functions changing their $k$-monotonicity at a certain number of points, or a class of functions having certain other shape characteristics on various subsets of $[-1,1]$, etc.

Recall that the Sobolev space $\mathbb{W}^{\nu}\left(\mathbb{L}_{p}\right)$ is a collection of all functions $f$ with an absolutely continuous $(\nu-1)$ st derivative such that $f^{(\nu)} \in \mathbb{L}_{p}[-1,1]$.

Theorem 4.1 (Constrained polynomial approximation). Let $n, m, \nu \in \mathbb{N}, \nu \leq m$, $f \in \mathbb{L}_{p}[-1,1] \cap \mathcal{M}, 0<p \leq \infty$, where $\mathcal{M}$ is some constraints class, and let $\mathcal{W}^{\nu}$ be some smoothness class of functions which is contained in the Sobolev space $\mathbb{W}^{\nu}\left(\mathbb{L}_{p}\right)$. Suppose that $\mathbf{z}_{n}$ is either a quasi-uniform or a Chebyshev partition of $[-1,1]$, and suppose also that the following assumptions are satisfied.

Assumption 1. For any $g \in \mathcal{W}^{\nu} \cap \mathcal{M}$ there exists a polynomial $q_{n} \in \Pi_{n} \cap \mathcal{M}$ such that

$$
\left\|g-q_{n}\right\|_{p} \leq c_{1} n^{-\nu} \mathrm{w}_{m-\nu}\left(g^{(\nu)}, n^{-1}\right)_{p} .
$$


Assumption 2. For some $r \geq m-1$, there exists a spline $s \in \mathcal{S}_{r}\left(\mathbf{z}_{n}\right) \cap \mathcal{M} \cap \mathcal{W}^{\nu}$ such that

$$
\|f-s\|_{p} \leq c_{2} \mathrm{w}_{m}\left(f, n^{-1}\right)_{p} .
$$

Then, there exists a polynomial $p_{n} \in \Pi_{n} \cap \mathcal{M}$ such that

$$
\left\|f-p_{n}\right\|_{p} \leq c \mathrm{w}_{m}\left(f, n^{-1}\right)_{p},
$$

where $c=c\left(c_{1}, c_{2}, r, m, \nu, p\right)$, and $c$ depends also on $\Delta$ in the case for a $\Delta$-quasiuniform partition $\mathbf{z}_{n}$.

Proof. If, by Assumption 2, $s \in \mathcal{S}_{r}\left(\mathbf{z}_{n}\right) \cap \mathcal{M} \cap \mathcal{W}^{\nu}$ is such that

$$
\|f-s\|_{p} \leq c \mathrm{w}_{m}\left(f, n^{-1}\right)_{p},
$$

we use Assumption 1 to conclude that there exists a polynomial $p_{n} \in \Pi_{n} \cap \mathcal{M}$ satisfying

$$
\left\|s-p_{n}\right\|_{p} \leq c n^{-\nu} \mathrm{w}_{m-\nu}\left(s^{(\nu)}, n^{-1}\right)_{p} .
$$

Therefore, using Theorems 1.4 and 1.8, we have

$$
\begin{aligned}
& \left\|f-p_{n}\right\|_{p} \leq c\|f-s\|_{p}+c\left\|s-p_{n}\right\|_{p} \\
& \quad \leq c\|f-s\|_{p}+c n^{-\nu} \mathrm{w}_{m-\nu}\left(s^{(\nu)}, n^{-1}\right)_{p} \leq c\|f-s\|_{p}+c \mathrm{w}_{m}\left(s, n^{-1}\right)_{p} \\
& \quad \leq c\|f-s\|_{p}+c \mathrm{w}_{m}\left(f, n^{-1}\right)_{p} \leq c \mathrm{w}_{m}\left(f, n^{-1}\right)_{p} .
\end{aligned}
$$

We can use the same idea to show how piecewise polynomials having "minimal" smoothness can be replaced by smoother splines.

Theorem 4.2 (Constrained approximation by smooth splines). Let $n, m, \nu, \mu \in \mathbb{N}$, $\nu \leq m, f \in \mathbb{L}_{p}[-1,1] \cap \mathcal{M}, 0<p \leq \infty$, where $\mathcal{M}$ is some constraints class, and let $\mathcal{W}^{\nu}$ be some smoothness class of functions which is contained in the Sobolev space $\mathbb{W}^{\nu}\left(\mathbb{L}_{p}\right)$. Suppose that $\mathbf{z}_{n}$ is either a quasi-uniform or a Chebyshev partition of $[-1,1]$, and suppose also that the following assumptions are satisfied.

Assumption 1. For any $g \in \mathcal{W}^{\nu} \cap \mathcal{M}$ and any $r \geq m-1$, there exists a spline $\widetilde{s} \in \mathcal{S}_{r}\left(\mathbf{z}_{n}\right) \cap \mathcal{M} \cap \mathbb{C}^{\mu}[-1,1]$ such that

$$
\|g-\widetilde{s}\|_{p} \leq c_{3} n^{-\nu} \mathrm{w}_{m-\nu}\left(g^{(\nu)}, n^{-1}\right)_{p} .
$$

Assumption 2. For some $r \geq m-1$, there exists a spline $s \in \mathcal{S}_{r}\left(\mathbf{z}_{n}\right) \cap \mathcal{M} \cap \mathcal{W}^{\nu}$ such that

$$
\|f-s\|_{p} \leq c_{4} \mathrm{w}_{m}\left(f, n^{-1}\right)_{p} .
$$

Then, for any $r \geq m-1$, there exists a spline $\mathbf{s}_{n} \in \mathcal{S}_{r}\left(\mathbf{z}_{n}\right) \cap \mathcal{M} \cap \mathbb{C}^{\mu}[-1,1]$ such that

$$
\left\|f-\mathbf{s}_{n}\right\|_{p} \leq c \mathrm{w}_{m}\left(f, n^{-1}\right)_{p},
$$

where $c=c\left(c_{3}, c_{4}, r, m, \nu, \mu, p\right)$, and $c$ depends also on $\Delta$ in the case for a $\Delta$-quasiuniform partition $\mathbf{z}_{n}$.

The proof is exactly the same as that of Theorem 4.1 and will be omitted.

Remark 4.3. Suppose that $f$ in Theorems 4.1 and 4.2 is assumed to be in $\mathbb{W}^{l}\left(\mathbb{L}_{p}\right)$ for some $0 \leq l<\nu$, and Assumptions 2 are replaced by the following. 
Assumption 2'. For some $r \geq m-1$, there exists a spline $s \in \mathcal{S}_{r}\left(\mathbf{z}_{n}\right) \cap \mathcal{N} \cap \mathcal{W}^{\nu}$ such that $\|f-s\|_{p} \leq c n^{-l} \mathrm{w}_{m-l}\left(f^{(l)}, n^{-1}\right)_{p}$. Then, there exists a polynomial $p_{n} \in \Pi_{n} \cap \mathcal{M}$ or a spline $\mathbf{s}_{n} \in \mathcal{S}_{r}\left(\mathbf{z}_{n}\right) \cap \mathcal{M} \cap \mathbb{C}^{\mu}[-1,1]$ such that their errors of approximation of $f$ are bounded by $c n^{-l} \mathrm{w}_{m-l}\left(f^{(l)}, n^{-1}\right)_{p}$.

The proof is exactly the same as that of Theorem 4.1

\section{Applications}

Given $k \geq 0$ and a set $I \subset \mathbb{R}$, a function $f$ is said to be $k$-monotone on $[-1,1]$ if its $k$ th divided differences $\left[x_{0}, \ldots, x_{k}\right] f$ are nonnegative for all choices of $(k+1)$ distinct points $x_{0}, \ldots, x_{k}$ in $[-1,1]$. We denote the class of all such functions by $\mathcal{M}^{k}$, and note that $\mathcal{M}^{1}$ and $\mathcal{M}^{2}$ are the collections of all nondecreasing and convex functions, respectively.

For a function $f \in \mathbb{L}_{p}[-1,1], 0<p \leq \infty$, we denote by

$$
E(f, \mathcal{F})_{p}:=\inf _{g \in \mathcal{F}}\|f-g\|_{p}
$$

the error of $\mathbb{L}_{p}$-approximation of $f$ by elements from the set $\mathcal{F} \subset \mathbb{L}_{p}[-1,1]$. In particular,

$$
\mathcal{E}_{r}^{(k)}\left(f, \mathbf{z}_{n}\right)_{p}:=E\left(f, \mathcal{S}_{r}\left(\mathbf{z}_{n}\right) \cap \mathcal{M}^{k}\right)_{p}
$$

and

$$
\widetilde{\mathcal{E}}_{r}^{(k)}\left(f, \mathbf{z}_{n}\right)_{p}:=E\left(f, \mathcal{S}_{r}\left(\mathbf{z}_{n}\right) \cap \mathcal{M}^{k} \cap \mathbb{C}^{r-1}[-1,1]\right)_{p}
$$

are the errors of $\mathbb{L}_{p}$-approximation of $f$ by splines from $\mathcal{S}_{r}\left(\mathbf{z}_{n}\right)$ and from $\mathcal{S}_{r}\left(\mathbf{z}_{n}\right) \cap$ $\mathbb{C}^{r-1}$ (i.e., having maximum smoothness) which are $k$-monotone on $[-1,1]$.

The following theorem basically says that if a certain Jackson type estimate is valid for all $\mathbb{C}^{\nu}$ functions, then it is also valid for $\mathbb{C}^{\nu-1}$ splines (i.e., if a function being approximated is a spline, then the smoothness requirement may be slightly relaxed).

Lemma 5.1. Let $\nu \in \mathbb{N}, m, r \in \mathbb{N}_{0}, 0<p \leq \infty$, and let $s \in \mathcal{S}_{r}\left(\mathbf{z}_{n}\right) \cap \mathcal{M}^{k}, k \in \mathbb{N}$, be in $\mathbb{C}^{\nu-1}[-1,1]$. Then, for any $\varepsilon>0$, there exists a function $g \in \mathbb{C}^{\nu}[-1,1] \cap \mathcal{M}^{k}$ such that

$$
\omega_{m}\left(g^{(\nu)}, n^{-1}\right)_{p} \leq c(m, r, \nu, p) \omega_{m}\left(s^{(\nu)}, n^{-1}\right)_{p}
$$

and

$$
\|g-s\|_{p} \leq \varepsilon .
$$

Moreover, if $0<p<\infty$, or $p=\infty$ and $\mathbf{z}_{n}=\mathbf{t}_{n}$, the following inequality is also satisfied:

$$
\omega_{m, \nu}^{\varphi}\left(g^{(\nu)}, n^{-1}\right)_{p} \leq c(m, r, \nu, p) \omega_{m, \nu}^{\varphi}\left(s^{(\nu)}, n^{-1}\right)_{p} .
$$

Proof. Since any function in $\mathcal{M}^{k}$ is necessarily in $\mathbb{C}^{k-2}(-1,1)$, a spline in $\mathcal{M}^{k}$ has to be in $\mathbb{C}^{k-2}[-1,1]$. Therefore, we can assume that $\nu \geq k-1$. Also, we can assume that $\omega_{m}\left(s^{(\nu)}, n^{-1}\right)_{p} \neq 0$ and $\omega_{m, \nu}^{\varphi}\left(s^{(\nu)}, n^{-1}\right)_{p} \neq 0$ (otherwise, $s$ is a polynomial and, hence, is certainly in $\mathbb{C}^{\nu}$ ).

Let $\delta>0$ be a small positive number to be determined later, and denote $\Delta s_{j}^{\nu}:=$ $s^{(\nu)}\left(z_{j}+\right)-s^{(\nu)}\left(z_{j}-\right), 1 \leq j \leq n-1$. For each $1 \leq j \leq n-1$ such that $\Delta s_{j}^{\nu} \neq 0$, we define $O_{j}:=\left[z_{j}-\delta, z_{j}\right]$ if $\Delta s_{j}^{\nu}>0$, and $O_{j}:=\left[z_{j}, z_{j}+\delta\right]$ if $\Delta s_{j}^{\nu}<0$. For $x \in O_{j}$, let $g^{(\nu)}$ be a liner polynomial interpolating $s^{(\nu)}$ at the endpoints of $O_{j}$. In other words, $g^{(\nu)}$ is the line through $\left(z_{j}-\delta, s^{(\nu)}\left(z_{j}-\delta\right)\right)$ and $\left(z_{j}, s^{(\nu)}\left(z_{j}+\right)\right)$ if $\Delta s_{j}^{\nu}>0$, and the 
line through $\left(z_{j}, s^{(\nu)}\left(z_{j}-\right)\right)$ and $\left(z_{j}+\delta, s^{(\nu)}\left(z_{j}+\delta\right)\right)$ if $\Delta s_{j}^{\nu}<0$. For all other $x$, we set $g^{(\nu)}(x):=s^{(\nu)}(x)$. We now define $g$ to be such that $g^{(l)}(-1)=s^{(l)}(-1)$ for all $0 \leq l \leq \nu-1$ and, hence,

$$
g(x)-s(x)=\frac{1}{(\nu-1) !} \int_{-1}^{x}(x-t)^{\nu-1}\left(g^{(\nu)}(t)-s^{(\nu)}(t)\right) d t, \quad x \in[-1,1] .
$$

Clearly, $g \in \mathbb{C}^{\nu}[-1,1]$. Also, since $s^{(\nu+1)}$ is bounded, it is possible to choose $\delta$ to be so small that $g^{(\nu)}(x) \geq s^{(\nu)}(x)$ for all $x \neq z_{j}$. It is also clear from the construction that, if $s^{(\nu)}$ is nondecreasing, then so is $g^{(\nu)}$. In particular, this implies that, if $\nu=k-1$ or $\nu=k$, then $g \in \mathcal{M}^{k}$. If $\nu \geq k+1$, we can use (5.4) and the inequality $g^{(\nu)} \geq s^{(\nu)}$ a.e. to conclude that $g^{(k)}(x) \geq s^{(k)}(x)$ for all $x \in[-1,1]$, and so $g \in \mathcal{M}^{k}$ in this case as well.

Since $\left\|g^{(\nu)}-s^{(\nu)}\right\|_{\mathbb{L}_{\infty}[-1,1]}$ is bounded and meas $\left\{x \mid g^{(\nu)}(x) \neq s^{(\nu)}(x)\right\} \rightarrow 0$ as $\delta \rightarrow 0$, we conclude that (5.2) is satisfied for sufficiently small $\delta>0$. Similarly (using the inequalities $\mathrm{w}\left(f_{1}+f_{2}, t\right)_{p} \leq c \mathrm{w}\left(f_{1}, t\right)_{p}+c \mathrm{w}\left(f_{2}, t\right)_{p}$ and $\mathrm{w}(f, t)_{p} \leq c\|f\|_{p}$, where $\mathrm{w}$ is $\omega_{m}$ or $\left.\omega_{m, \nu}^{\varphi}\right)$, (5.1) and (5.3) are valid for sufficiently small $\delta>0$ if $0<p<\infty$, since $\left\|g^{(\nu)}-s^{(\nu)}\right\|_{p} \rightarrow 0$ as $\delta \rightarrow 0$.

Hence, it remains to prove (5.1) and (5.3) in the case $p=\infty$. Assume that $\delta>0$ is so small that $\left\|s^{(\nu)}-g^{(\nu)}\right\|_{\mathbb{L}_{\infty}\left(O_{j}\right)} \leq 2\left|\Delta s_{j}^{\nu}\right|$ for all $j$ such that $\Delta s_{j}^{\nu} \neq 0(\operatorname{such} \delta$ exists since $s^{(\nu)}$ is continuous to the left and to the right of $z_{j}$ ). Since it immediately follows from the definition that $\left|\Delta s_{j}^{\nu}\right| \leq \omega_{m}\left(s^{(\nu)}, n^{-1}\right)_{\infty}$ for all $1 \leq j \leq n-1$, this implies that

Therefore,

$$
\left\|s^{(\nu)}-g^{(\nu)}\right\|_{\infty} \leq 2 \omega_{m}\left(s^{(\nu)}, n^{-1}\right)_{\infty}
$$

$$
\omega_{m}\left(g^{(\nu)}, n^{-1}\right)_{\infty} \leq c\left\|s^{(\nu)}-g^{(\nu)}\right\|_{\infty}+\omega_{m}\left(s^{(\nu)}, n^{-1}\right)_{\infty} \leq c \omega_{m}\left(s^{(\nu)}, n^{-1}\right)_{\infty},
$$

and (5.1) is proved.

To prove (5.3) for $p=\infty$ and $\mathbf{z}_{n}=\mathbf{t}_{n}$, note that, by the definition, $\varphi\left(z_{j}\right)^{\nu} \Delta s_{j}^{\nu} \leq$ $\omega_{m, \nu}^{\varphi}\left(s^{(\nu)}, n^{-1}\right)_{\infty}$. Suppose now that $x_{0} \in[-1,1]$ and $0<h_{0} \leq n^{-1}$ are such that

$$
\omega_{m, \nu}^{\varphi}\left(g^{(\nu)}, n^{-1}\right)_{\infty} \leq 2 \varphi\left(x_{0}\right)^{\nu}\left|\Delta_{h_{0} \varphi\left(x_{0}\right)}^{m}\left(g^{(\nu)}, x_{0}\right)\right| .
$$

Then, denoting $I\left(x_{0}, h_{0}, m\right):=\left[x_{0}-m h_{0} \varphi\left(x_{0}\right) / 2, x_{0}+m h_{0} \varphi\left(x_{0}\right) / 2\right]$ and taking into account that $\varphi\left(x_{0}\right) \leq c(m) \varphi\left(z_{j}\right)$, for all $z_{j} \in I\left(x_{0}, h_{0}, m\right.$ ) (in fact, we do not need the condition $\mathbf{z}_{n}=\mathbf{t}_{n}$ for this inequality to hold, it'd be sufficient to require that $\min \left\{1-z_{n-1}, 1+z_{1}\right\} \geq c n^{-2}$ ), we have

$$
\begin{aligned}
& \omega_{m, \nu}^{\varphi}\left(g^{(\nu)}, n^{-1}\right)_{\infty} \\
& \quad \leq c \varphi\left(x_{0}\right)^{\nu}\left|\Delta_{h_{0} \varphi\left(x_{0}\right)}^{m}\left(s^{(\nu)}, x_{0}\right)\right|+c \varphi\left(x_{0}\right)^{\nu}\left\|g^{(\nu)}-s^{(\nu)}\right\|_{\mathbb{L}_{\infty}\left(I\left(x_{0}, h_{0}, m\right)\right)} \\
& \quad \leq c \omega_{m, \nu}^{\varphi}\left(s^{(\nu)}, n^{-1}\right)_{\infty}+c \varphi\left(x_{0}\right)^{\nu} \max \left\{\Delta s_{j}^{\nu} \mid 1 \leq j \leq n-1, z_{j} \in I\left(x_{0}, h_{0}, m\right)\right\} \\
& \quad \leq c \omega_{m, \nu}^{\varphi}\left(s^{(\nu)}, n^{-1}\right)_{\infty},
\end{aligned}
$$

and the proof is complete.

Taking into account Lemma 5.1, Theorems 4.1 and 4.2 can now be restated for uniform partitions and $\mathcal{M}:=\mathcal{M}^{k}, k \in \mathbb{N}$, as follows (note that we combined both statements into one). We also remark that analogs of Corollary 5.2 are certainly 
valid for other kinds of partitions (quasi-uniform and Chebyshev) as well, and we only state it here for the uniform partition for simplicity.

Corollary 5.2. Let $k, m, n, \nu \in \mathbb{N}$ be such that $m \geq \nu$, and let $f \in \mathbb{L}_{p}[-1,1] \cap \mathcal{M}^{k}$, $0<p \leq \infty$. Suppose that $\mathbf{u}_{n}$ is a uniform partition of $[-1,1], \mathcal{K}$ is either $\Pi_{n}$ or $\mathcal{S}_{r}\left(\mathbf{u}_{n}\right) \cap \mathbb{C}^{\mu}[-1,1]$, and the following assumptions are satisfied.

Assumption 1. For any $g \in \mathbb{C}^{\nu}[-1,1] \cap \mathcal{M}^{k}$,

$$
E\left(g, \mathcal{K} \cap \mathcal{M}^{k}\right)_{p} \leq c_{5} n^{-\nu} \omega_{m-\nu}\left(g^{(\nu)}, n^{-1}\right)_{p} .
$$

Assumption 2. For some $r \in \mathbb{N}, r \geq m-1$,

$$
E\left(f, \mathcal{S}_{r}\left(\mathbf{u}_{n}\right) \cap \mathbb{C}^{\nu-1}[-1,1] \cap \mathcal{M}^{k}\right)_{p} \leq c_{6} \omega_{m}\left(f, n^{-1}\right)_{p} .
$$

Then,

$$
E\left(f, \mathcal{K} \cap \mathcal{M}^{k}\right)_{p} \leq c \omega_{m}\left(f, n^{-1}\right)_{p},
$$

where $c=c\left(c_{5}, c_{6}, r, m, \nu, p\right)$, and $c$ depends also on $\mu$ if $\mathcal{K}=\mathcal{S}_{r}\left(\mathbf{u}_{n}\right) \cap \mathbb{C}^{\mu}[-1,1]$.

5.1. Monotone and convex spline approximation. The following corollary states that, for any monotone function $f$, there exists a spline of whatever smoothness we need whose order of approximation is bounded by $c \omega_{2}\left(f, n^{-1}\right)_{p}$. It is well known that $\omega_{2}$ cannot be replaced by $\omega_{3}$ (see [19]). While this theorem is known for splines of small degrees, it is new for arbitrary $r$.

Corollary 5.3. For $f \in \mathbb{L}_{p}[-1,1] \cap \mathcal{M}^{1}, 1 \leq p \leq \infty$, and any $r \in \mathbb{N}$,

$$
\widetilde{\mathcal{E}}_{r}^{(1)}\left(f, \mathbf{u}_{n}\right)_{p} \leq c \omega_{2}\left(f, n^{-1}\right)_{p} .
$$

Proof. It was shown by Chui, Smith and Ward [3] (see also DeVore [5] in the case $p=\infty)$ that, for $g \in \mathbb{W}^{1}\left(\mathbb{L}_{p}\right) \cap \mathcal{M}^{1}, 1 \leq p \leq \infty$, and $r \in \mathbb{N}$,

$$
\widetilde{\mathcal{E}}_{r}^{(1)}\left(g, \mathbf{u}_{n}\right)_{p} \leq c n^{-1} \omega\left(g^{\prime}, n^{-1}\right)_{p} .
$$

It is well known that there is a spline $s \in \mathcal{S}_{1}\left(\mathbf{u}_{n}\right) \cap \mathbb{C}[-1,1] \cap \mathcal{M}^{1}$ such that

$$
\|s-f\|_{p} \leq c \omega_{2}\left(f, n^{-1}\right)_{p} .
$$

(In the case $p=\infty$, we can simply take a piecewise linear function interpolating $f$ at the knots in $\mathbf{u}_{n}$. If $p<\infty$, one can use the same argument as in [6], for example.) Finally, Corollary 5.2 with $k=1, \nu=1, m=2, \mu=r-1$, and $\mathcal{K}=\mathcal{S}_{r}\left(\mathbf{u}_{n}\right) \cap \mathbb{C}^{r-1}[-1,1]$ completes the proof.

The following result is an analog of Corollary 5.3 for convex spline approximation in $\mathbb{L}_{\infty}[-1,1]$. It follows from [19] that $\omega_{3}$ in (5.5) cannot be replaced by $\omega_{4}$. The author is not aware of analogs of (5.6) in the $\mathbb{L}_{p}$ metric for $p<\infty$ (except for the cases $r=2$ and $r=3$ which were considered in [6]). Hence, it is still unresolved if Corollary 5.4 is valid with $\mathbb{L}_{p}, p<\infty$, replacing $\mathbb{L}_{\infty}$ everywhere in its statement.

Corollary 5.4. For $f \in \mathbb{C}[-1,1] \cap \mathcal{M}^{2}$ and any $r \geq 2$,

$$
\widetilde{\mathcal{E}}_{r}^{(2)}\left(f, \mathbf{u}_{n}\right)_{\infty} \leq c \omega_{3}\left(f, n^{-1}\right)_{\infty} .
$$

Proof. It follows from a result of Beatson [1] that, for $g \in \mathbb{C}^{2}[-1,1] \cap \mathcal{M}^{2}$ and $r \geq 2$,

$$
\widetilde{\mathcal{E}}_{r}^{(2)}\left(g, \mathbf{u}_{n}\right)_{\infty} \leq c(r) n^{-2} \omega\left(f^{\prime \prime}, n^{-1}\right)_{\infty} .
$$

Ivanov and Popov [13] proved that, for a convex function $f$ on $[-1,1]$,

$$
\widetilde{\varepsilon}_{2}^{(2)}\left(f, \mathbf{u}_{n}\right)_{\infty} \leq c \omega_{3}\left(f, n^{-1}\right)_{\infty} .
$$


Now, Corollary 5.2 with $k=2, \nu=2, m=3, \mu=r-1$, and $\mathcal{K}=\mathcal{S}_{r}\left(\mathbf{u}_{n}\right) \cap$ $\mathbb{C}^{r-1}[-1,1]$ completes the proof.

\subsection{Monotone and convex polynomial approximation.}

Corollary 5.5 (Shevchuk [18). For $f \in \mathbb{C}^{1}[-1,1] \cap \mathcal{M}^{1}, r \in \mathbb{N}$ and $n \geq r$,

$$
E\left(f, \Pi_{n} \cap \mathcal{M}^{1}\right)_{\infty} \leq c(r) n^{-1} \omega_{r}\left(f^{\prime}, n^{-1}\right)_{\infty} .
$$

Note that pointwise estimates (which are stronger than (5.8)) were obtained in 18 .

Proof. It was shown by Leviatan and Mhaskar [15] that, for $g \in \mathbb{C}^{1}[-1,1] \cap \mathcal{M}^{1}$ and $r \in \mathbb{N}$,

$$
\widetilde{\mathcal{E}}_{r+1}^{(1)}\left(g, \mathbf{u}_{n}\right)_{\infty} \leq c n^{-1} \omega_{r+1}\left(g^{\prime}, n^{-1}\right)_{\infty} .
$$

Also, DeVore 4] proved that for any $g \in \mathbb{C}^{r}[-1,1] \cap \mathcal{M}^{1}$,

$$
E\left(g, \Pi_{n} \cap \mathcal{M}^{1}\right)_{\infty} \leq c(r) n^{-r} \omega\left(f^{(r)}, n^{-1}\right)_{\infty} .
$$

Now, the same proof as that of Theorem 4.1 (see also Remark 4.3) completes the proof of the corollary.

Corollary 5.6 (Hu, Leviatan and $\mathrm{Yu}[11])$. If $f \in \mathbb{C}[-1,1] \cap \mathcal{M}^{2}$ and $n \geq 2$, then

$$
E\left(f, \Pi_{n} \cap \mathcal{M}^{2}\right)_{\infty} \leq c \omega_{3}\left(f, n^{-1}\right)_{\infty} .
$$

Proof. It follows from a result of Manya (see [18, Theorem 17.2]) that, for $g \in$ $\mathbb{C}^{2}[-1,1] \cap \mathcal{M}^{2}$ and $n \geq 2$,

$$
E\left(g, \Pi_{n} \cap \mathcal{M}^{2}\right)_{\infty} \leq c n^{-2} \omega\left(f^{\prime \prime}, n^{-1}\right)_{\infty} .
$$

Now, the estimate (5.7) and Corollary 5.2 with $k=2, \nu=2, m=3$, and $\mathcal{K}=\Pi_{n}$ complete the proof.

Corollary 5.7. If $f \in \mathbb{L}_{p}[-1,1] \cap \mathcal{M}^{2}, 1 \leq p<\infty$ and $n \geq 2$, then

$$
E\left(f, \Pi_{n} \cap \mathcal{M}^{2}\right)_{p} \leq c \omega_{3}\left(f, n^{-1}\right)_{p} .
$$

Proof. It was shown in [14] that for any $g \in \mathbb{C}^{1}[-1,1] \cap \mathcal{M}^{2}$ and $n \geq 2$,

$$
E\left(f, \Pi_{n} \cap \mathcal{M}^{2}\right)_{p} \leq c n^{-1} \omega_{2}\left(f^{\prime}, n^{-1}\right)_{p}, \quad 1 \leq p<\infty .
$$

Now, the estimate $\widetilde{\mathcal{E}}_{2}^{(2)}\left(g, \mathbf{u}_{n}\right)_{p} \leq c \omega_{3}\left(g, n^{-1}\right)_{p}$, for every convex $g \in \mathbb{L}_{p}[-1,1]$ (see [6. Theorem 2.3]), and Corollary [5.2 with $k=2, \nu=1, m=3$, and $\mathcal{K}=\Pi_{n}$ complete the proof.

In order to use Corollary 5.2 to obtain an estimate in terms of $\omega_{3}^{\varphi}\left(f, n^{-1}\right)_{\infty}$ for convex polynomial approximation (established in 14 with a rather long and technical proof) we would need a convex $\mathbb{C}^{4}$ spline on a Chebyshev partition yielding an estimate in terms of $\omega_{3}^{\varphi}\left(f, n^{-1}\right)_{\infty}$. At present, the author is not aware of any such results. Hence, it is an open problem to prove analogs of Corollaries 5.3 and 5.4 for splines on Chebyshev partitions, as well as to investigate the validity of other Jackson type estimates involving moduli of derivatives of functions. The following has been partially resolved only in some cases (for example, polynomial constrained approximation in the case $p=\infty$ ). 
Open Problem 5.8. Let $k=1$ or 2 , and $0<p \leq \infty$. For each $\nu \in \mathbb{N}_{0}$, determine all $m \in \mathbb{N}_{0}$ so that for all $f \in \mathbb{C}^{\nu}[-1,1] \cap \mathcal{M}^{k}$,

$$
\widetilde{\mathcal{E}}_{r}^{(k)}\left(f, \mathbf{t}_{n}\right)_{p} \leq c(m, \nu) n^{-\nu} \omega_{m, \nu}^{\varphi}\left(f^{(\nu)}, n^{-1}\right)_{p}, \quad r \geq m+\nu-1,
$$

or

$$
E\left(f, \Pi_{n} \cap \mathcal{M}^{k}\right)_{p} \leq c(m, \nu) n^{-\nu} \omega_{m, \nu}^{\varphi}\left(f^{(\nu)}, n^{-1}\right)_{p}, \quad n \geq m+\nu-1 .
$$

6. Appendix: Properties of $\omega_{k, m}^{\varphi}(f, t)_{p}$ Moduli

Surprisingly, the following theorem seems to be new. Note that the inequality [9, Corollary 6.3.3 (b)] cannot be used with $\varphi=\sqrt{1-x^{2}}$.

Theorem 6.1. For $k, \nu \in \mathbb{N}$ and $f \in \mathbb{W}^{\nu}\left(\mathbb{L}_{p}\right), 1 \leq p \leq \infty$,

$$
\omega_{k+\nu}^{\varphi}(f, t)_{p} \leq c(k, \nu) t^{\nu} \omega_{k, \nu}^{\varphi}\left(f^{(\nu)}, t\right)_{p} .
$$

Proof. We only consider the case $p<\infty$ since considerations for $p=\infty$ are much simpler. Let $n \geq(k+\nu) / 2$ and suppose that $\mathbf{t}_{n}$ is a Chebyshev partition. Recall (see Section 2) that $\mathfrak{I}_{j, k+\nu}=\left[t_{j-3(k+\nu)}, t_{j+4+3(k+\nu)}\right]$, denote $\mathfrak{I}_{j}:=\mathfrak{I}_{j, k+\nu}$, and for each $0 \leq j \leq n-1$, let $q_{j} \in \Pi_{k+\nu-1}$ be such that $\left\|f-q_{j}\right\|_{\mathbb{L}_{p}\left(\mathfrak{I}_{j}\right)} \leq c \omega_{k+\nu}\left(f, \mathfrak{I}_{j}\right)_{p}$ ( $q_{j}$ exists by Lemma 2.1). Then,

$$
\begin{aligned}
& \omega_{k+\nu}^{\varphi}\left(f, n^{-1}\right)_{p}^{p}=\sup _{0<h \leq n^{-1}}\left\|\Delta_{h \varphi}^{k+\nu}(f, \cdot)\right\|_{p}^{p} \\
& =\sup _{0<h \leq n^{-1}} \sum_{j=0}^{n-1} \int_{J_{j}}\left|\Delta_{h \varphi(x)}^{k+\nu}\left(f-q_{j}, x\right)\right|^{p} d x \\
& \leq c \sup _{0<h \leq n^{-1}} \sum_{j=0}^{n-1} \sum_{i=0}^{k+\nu} \int_{J_{j} \cap \mathfrak{D}_{(k+\nu) h}}\left|\left(f-q_{j}\right)(x+(i-(k+\nu) / 2) h \varphi(x))\right|^{p} d x .
\end{aligned}
$$

Now, since $x+(i-(k+\nu) / 2) h \varphi(x) \in \mathfrak{I}_{j}$ and, as can be verified by straightforward computations, $\frac{d}{d x}(x+(i-(k+\nu) / 2) h \varphi(x)) \geq 1 / 2$, for all $x \in J_{j} \cap \mathfrak{D}_{(k+\nu) h}$ and $0<h \leq n^{-1}$ (provided that $n$ is sufficiently large, $n \geq(k+\nu) / 2$ will do), using the substitution $y=x+(i-(k+\nu) / 2) h \varphi(x)$ we get

$$
\begin{aligned}
\omega_{k+\nu}^{\varphi}\left(f, n^{-1}\right)_{p}^{p} & \leq c \sum_{j=0}^{n-1}\left\|f-q_{j}\right\|_{\mathbb{L}_{p}\left(\mathfrak{I}_{j}\right)}^{p} \leq c \sum_{j=0}^{n-1} \omega_{k+\nu}\left(f, \mathfrak{I}_{j}\right)_{p}^{p} \\
& \leq c \sum_{j=0}^{n-1}\left|\mathfrak{I}_{j}\right|^{-1} \int_{0}^{\left|\mathfrak{I}_{j}\right|} \int_{\mathfrak{I}_{j}}\left|\Delta_{h}^{k+\nu}(f, x)\right|^{p} d x d h
\end{aligned}
$$

where the last inequality follows from (2.4). Now, taking into account the identity

$$
\Delta_{h}^{k+1}(f, x)=\int_{-h / 2}^{h / 2} \Delta_{h}^{k}\left(f^{\prime}, x+u\right) d u,
$$

which implies

$$
\Delta_{h}^{k+\nu}(f, x)=\int_{-h / 2}^{h / 2} \ldots \int_{-h / 2}^{h / 2} \Delta_{h}^{k}\left(f^{(\nu)}, x+u_{1}+\cdots+u_{\nu}\right) d u_{1} \ldots d u_{\nu},
$$


and using Hölder's inequality we have

$$
\begin{aligned}
\omega_{k+\nu}^{\varphi}\left(f, n^{-1}\right)_{p}^{p} \leq & c \sum_{j=0}^{n-1}\left|\mathfrak{I}_{j}\right|^{-1} \int_{0}^{\left|\mathfrak{I}_{j}\right|} \int_{\mathfrak{I}_{j}} h^{(p-1) \nu} \int_{-h / 2}^{h / 2} \ldots \int_{-h / 2}^{h / 2} \\
& \times\left|\Delta_{h}^{k}\left(f^{(\nu)}, x+u_{1}+\cdots+u_{\nu}\right)\right|^{p} d u_{1} \ldots d u_{\nu} d x d h \\
\leq & c \sum_{j=0}^{n-1}\left|\mathfrak{I}_{j}\right|^{-1} \int_{0}^{\left|\mathfrak{I}_{j}\right|} h^{(p-1) \nu} \int_{-h / 2}^{h / 2} \ldots \int_{-h / 2}^{h / 2} \int_{\mathfrak{I}_{j}} \\
& \times\left|\Delta_{h}^{k}\left(f^{(\nu)}, x+u_{1}+\cdots+u_{\nu}\right)\right|^{p} d x d u_{1} \ldots d u_{\nu} d h \\
\leq & c \sum_{j=0}^{n-1}\left|\mathfrak{I}_{j}\right|^{-1} \int_{0}^{\left|\mathfrak{I}_{j}\right|} h^{(p-1) \nu} \int_{-h / 2}^{h / 2} \ldots \int_{-h / 2}^{h / 2} \int_{\mathfrak{I}_{j}+u_{1}+\cdots+u_{\nu}} \\
& \times\left|\Delta_{h}^{k}\left(f^{(\nu)}, x\right)\right|^{p} d x d u_{1} \ldots d u_{\nu} d h .
\end{aligned}
$$

For $\mathfrak{I}_{j}=[a, b]$, we now denote $\tilde{\mathfrak{I}}_{j}:=\left[a-\nu\left|\mathfrak{I}_{j}\right| / 2, b+\nu\left|\mathfrak{I}_{j}\right| / 2\right] \cap[-1,1]$, and note that $\mathfrak{I}_{j}+u_{1}+\cdots+u_{\nu} \cap[-1+k h / 2,1-k h / 2] \subset \tilde{\mathfrak{I}}_{j}$ for $u_{i} \in[-h / 2, h / 2], 1 \leq i \leq \nu$, and $0<h \leq\left|\mathfrak{I}_{j}\right|$. Therefore,

$$
\begin{aligned}
& \omega_{k+\nu}^{\varphi}\left(f, n^{-1}\right)_{p}^{p} \leq c \sum_{j=0}^{n-1}\left|\mathfrak{I}_{j}\right|^{-1} \int_{0}^{\left|\mathfrak{I}_{j}\right|} h^{p \nu} \int_{\tilde{\mathfrak{I}}_{j}}\left|\Delta_{h}^{k}\left(f^{(\nu)}, x\right)\right|^{p} d x d h \\
& \leq c \sum_{j=0}^{n-1}\left|\mathfrak{I}_{j}\right|^{-1} \int_{\tilde{\mathfrak{I}}_{j}} \int_{0}^{\left|\mathfrak{I}_{j}\right| / \varphi(x)} \varphi(x)^{p \nu+1} h^{p \nu}\left|\Delta_{h \varphi(x)}^{k}\left(f^{(\nu)}, x\right)\right|^{p} d h d x .
\end{aligned}
$$

Now, $\left|\mathfrak{I}_{j}\right| / \varphi(x) \sim n^{-1}$ for all $x \in \tilde{\mathfrak{I}}_{j} \backslash\left(J_{0} \cup J_{n-1}\right)$. If $x \in\left(J_{0} \cup J_{n-1}\right) \cap \mathfrak{D}_{k h}$, then $4 k h /\left(4+k^{2} h^{2}\right) \leq \varphi(x) \leq \sin \left(\pi n^{-1}\right)$, which can only happen if $h \leq(8 / k) n^{-1}$. Therefore,

$$
\begin{aligned}
& \omega_{k+\nu}^{\varphi}\left(f, n^{-1}\right)_{p}^{p} \leq c \sum_{j=0}^{n-1} \int_{\tilde{\mathfrak{I}}_{j}} \int_{0}^{c n^{-1}} \varphi(x)\left|\mathfrak{I}_{j}\right|^{-1} h^{p \nu}\left|\varphi(x)^{\nu} \Delta_{h \varphi(x)}^{k}\left(f^{(\nu)}, x\right)\right|^{p} d h d x \\
& \leq c \int_{0}^{c n^{-1}} n h^{p \nu}\left\|\varphi^{\nu} \Delta_{h \varphi}^{k}\left(f^{(\nu)}, \cdot\right)\right\|_{p}^{p} d h \leq c n^{-p \nu} \omega_{k, \nu}^{\varphi}\left(f^{(\nu)}, c n^{-1}\right)_{p}^{p} .
\end{aligned}
$$

Hence, the inequality

$$
\omega_{k+\nu}^{\varphi}\left(f, n^{-1}\right)_{p} \leq c n^{-\nu} \omega_{k, \nu}^{\varphi}\left(f^{(\nu)}, c_{7} n^{-1}\right)_{p}
$$

is proved for all $n \geq(k+\nu) / 2$ (and without loss of generality we can assume that $\left.c_{7} \geq 1\right)$.

Now, given $0<t \leq 2 /(k+\nu)$ (for $t>2 /(k+\nu)$, we use the fact that $\left.\omega_{k+\nu}^{\varphi}(f, t)_{p}=\omega_{k+\nu}^{\varphi}(f, 2 /(k+\nu))_{p}\right)$, we let $n \geq(k+\nu) / 2$ be such that $c_{7} n^{-1} \leq t<$ $2 c_{7} n^{-1}$ (there may be more than one $n$ ), and using the inequality $\omega_{k+\nu}^{\varphi}(f, \lambda t)_{p} \leq$ $c(\lambda+1)^{k+\nu} \omega_{k+\nu}^{\varphi}(f, t)_{p}$ (see [9, Theorem 4.1.2]), we obtain

$$
\begin{aligned}
\omega_{k+\nu}^{\varphi}(f, t)_{p} & \leq \omega_{k+\nu}^{\varphi}\left(f, 2 c_{7} n^{-1}\right)_{p} \leq c \omega_{k+\nu}^{\varphi}\left(f, n^{-1}\right)_{p} \\
& \leq c n^{-\nu} \omega_{k, \nu}^{\varphi}\left(f^{(\nu)}, c_{7} n^{-1}\right)_{p} \leq c t^{\nu} \omega_{k, \nu}^{\varphi}\left(f^{(\nu)}, t\right)_{p},
\end{aligned}
$$

and the proof is now complete. 


\section{REFERENCES}

[1] R. K. Beatson, Convex approximation by splines, SIAM J. Math. Anal. 12 (1981), no. 4, 549-559. MR617714 (82h:41012)

[2] P. Borwein and T. Erdélyi, Polynomials and polynomial inequalities, Graduate Texts in Mathematics, vol. 161, Springer-Verlag, New York, 1995. MR1367960 (97e:41001)

[3] C. K. Chui, P. W. Smith, and J. D. Ward, Degree of $L_{p}$ approximation by monotone splines, SIAM J. Math. Anal. 11 (1980), no. 3, 436-447. MR572194 (81h:41019)

[4] R. A. DeVore, Monotone approximation by polynomials, SIAM J. Math. Anal. 8 (1977), no. 5, 906-921. MR0510582 (58:23252)

[5] _ Monotone approximation by splines, SIAM J. Math. Anal. 8 (1977), no. 5, 891-905. MR0510725 (58:23259)

[6] R. A. DeVore, Y. K. Hu, and D. Leviatan, Convex polynomial and spline approximation in $L_{p}, 0<p<\infty$, Constr. Approx. 12 (1996), no. 3, 409-422. MR1405006 (97j:41008)

[7] R. A. DeVore, D. Leviatan, and X. M. Yu, Polynomial approximation in $L_{p}(0<p<1)$, Constr. Approx. 8 (1992), no. 2, 187-201. MR1152876 (93f:41011)

[8] R. A. DeVore and G. G. Lorentz, Constructive approximation, Grundlehren der Mathematischen Wissenschaften [Fundamental Principles of Mathematical Sciences], vol. 303, SpringerVerlag, Berlin, 1993. MR.95f:41001

[9] Z. Ditzian and V. Totik, Moduli of smoothness, Springer Series in Computational Mathematics, vol. 9, Springer-Verlag, New York, 1987. MR0914149 (89h:41002)

[10] Y. Hu, On equivalence of moduli of smoothness, J. Approx. Theory 97 (1999), no. 2, 282-293. MR.1682954 (2000a:41044)

[11] Y. Hu, D. Leviatan, and X.M. Yu, Convex polynomial and spline approximation in $C[-1,1]$, Constr. Approx. 10 (1994), no. 1, 31-64. MR.1260358 (95a:41018)

[12] Y. Hu and X. M. Yu, Discrete modulus of smoothness of splines with equally spaced knots, SIAM J. Numer. Anal. 32 (1995), no. 5, 1428-1435. MR.1352197 (96h:41010)

[13] K. G. Ivanov and B. Popov, On convex approximation by quadratic splines, J. Approx. Theory 85 (1996), no. 1, 110-114. MR1382054 (97c:41024)

[14] K. A. Kopotun, Pointwise and uniform estimates for convex approximation of functions by algebraic polynomials, Constr. Approx. 10 (1994), no. 2, 153-178. MR1305916 (95k:41014)

[15] D. Leviatan and H. N. Mhaskar, The rate of monotone spline approximation in the $L_{p}$-norm, SIAM J. Math. Anal. 13 (1982), no. 5, 866-874. MR668327 (83j:41014)

[16] P. P. Petrushev and V. A. Popov, Rational approximation of real functions, Encyclopedia of Mathematics and its Applications, vol. 28, Cambridge University Press, Cambridge, 1987. MR940242 (89i:41022)

[17] I. A. Shevchuk, Approximation by Polynomials and Traces of the Functions Continuous on an Interval, Naukova Dumka, Kyiv, 1992.

[18] _ On co-approximation of monotone functions, Dokl. Akad. Nauk SSSR 308 (1989), no. 3, 537-541 (Russian). MR.1021110 (91c:41050)

[19] A. S. Švedov, Orders of coapproximation of functions by algebraic polynomials, Mat. Zametki 29 (1981), no. 1, 117-130, 156 (Russian). MR0604156 (82c:41009)

Department of Mathematics, University of Manitoba, Winnipeg, Manitoba, R3T 2N2, CANADA

E-mail address: kopotunk@cc.umanitoba.ca 